\title{
Measurement of the dependence of transverse energy production at large pseudorapidity on the hard scattering kinematics of proton-proton collisions at $\sqrt{s}=2.76 \mathrm{TeV}$ with the ATLAS detector
}

Peter Steinberg, on behalf of the ATLAS Collaboration

Brookhaven National Laboratory, Upton, NY 11973

\begin{abstract}
The relationship between jet production in the central region and the underlying event activity in a pseudorapidityseparated region is studied in $4.0 \mathrm{pb}^{-1}$ of $\sqrt{s}=2.76 \mathrm{TeV} p p$ events recorded with the ATLAS detector at the LHC. The hard scattering is characterised by the average transverse momentum and pseudorapidity of the two highest transverse momentum jets in the event. Results are also presented as a function of the scaled longitudinal momenta of the hardscattered partons in the target and projectile beam-protons. Transverse energy production at large pseudorapidity is observed to vary strongly with the longitudinal momentum fraction in the target proton and only weakly with that in the projectile proton.
\end{abstract}

Keywords:

\section{Introduction}

Measurements of the underlying event in protonproton collisions, especially at large rapidity are important to interpret recent results on jet production in proton-lead $(p+\mathrm{Pb})$ collisions $[1,2]$. In these results, single and dijet production in the proton-going (forward, or projectile) direction was found to have a significant dependence on the underlying event activity in the nucleus-going (backward, or target) direction that contradicts geometric models of how jet and underlying event production should correlate.

These results have several competing interpretations: Ref. [3] argues that they are evidence that proton configurations with a parton carrying a large fraction $x$ of the proton longitudinal momentum interact with a significantly smaller than average cross-section. Alternately, Refs. [4] and [5] have argued that in the constituent nucleon-nucleon $(N N)$ collisions, energy production at backward rapidities naturally decreases with increasing $x$ in the forward-going proton, either through the suppression of soft gluons available for particle production or from energy-momentum conservation between the hard process and soft particle production. More generally, the modification of soft particle production in $N N$ collisions in the presence of a hard process is expected to affect estimates of the collision geometry of $p+\mathrm{Pb}$ collisions with a hard scattering. Thus a control measurement in $p p$ collisions to determine how soft particle production at negative pseudorapidities varies with the $x$ in the projectile (target) beam-proton headed towards positive (negative) rapidity might provide insight into the relevance of the different scenarios.

This proceedings presents a measurement of the mean of the transverse energy at large pseudorapidity $\left(\left\langle\sum E_{\mathrm{T}}\right\rangle\right)$ in the direction of one of the protons in $p p$ collisions, as a function of the hard scattering kinematics in dijet events. For each kinematic selection, $\left\langle\sum E_{\mathrm{T}}\right\rangle$ is the average of the $\sum E_{\mathrm{T}}$ distribution in the selected events. $\sum E_{\mathrm{T}}$ is measured only on one side of the forward calorimeter. This is done in analogy with the centrality definition in $p+\mathrm{Pb}$ collisions [1] which is characterized by the $\sum E_{\mathrm{T}}$ in the forward calorimeter module 
situated at $-4.9<\eta<-3.2$, in the nucleus-going direction. In $p p$ collisions the asymmetric choice of the $\sum E_{\mathrm{T}}$ measuring region means that the target proton represents the role of one of the nucleons in the $\mathrm{Pb}$ nucleus. The results here are presented in more detail in Ref. [6].

Jets are reconstructed from local energy deposits in the calorimeter using the anti- $k_{t}$ algorithm [7] with radius parameter $R=0.4$. The quantity $\sum E_{\mathrm{T}}$ is measured by summing the transverse energy of the cells in the forward calorimeters. Equivalent definitions for jets and $\sum E_{\mathrm{T}}$ at the level of particles produced in event generators are described below. $\left\langle\sum E_{\mathrm{T}}\right\rangle$ is first reported as a function of the average transverse momentum, $p_{\mathrm{T}}^{\text {avg }}=\left(p_{\mathrm{T}, 1}+p_{\mathrm{T}, 2}\right) / 2$, and average pseudorapidity $\eta^{\text {dijet }}=\left(\eta_{1}+\eta_{2}\right) / 2$, of the leading and subleading jets in events.

Results are also reported as a function of two kinematic quantities $x_{\text {proj }}=p_{\mathrm{T}}^{\text {avg }}\left(e^{+\eta_{1}}+e^{+\eta_{2}}\right) / \sqrt{s}$ and $x_{\text {targ }}=$ $p_{\mathrm{T}}^{\text {avg }}\left(e^{-\eta_{1}}+e^{-\eta_{2}}\right) / \sqrt{s}$. In a leading-order approach, $x_{\text {proj }}$ approximates the Bjorken- $x$ of the hard-scattered parton in the beam-proton with positive rapidity, while $x_{\text {targ }}$ is for the other proton. Similar estimates have been used previously in dijet measurements at the CERN $\mathrm{S} p \bar{p} \mathrm{~S}$ collider [8]. Finally, to better reveal the relative dependence of $\left\langle\sum E_{\mathrm{T}}\right\rangle$ on the hard scattering kinematics, results are also reported as a ratio to a reference value $\left\langle\sum E_{\mathrm{T}}\right\rangle^{\text {ref }}$, which is the $\left\langle\sum E_{\mathrm{T}}\right\rangle$ evaluated at a fixed choice of dijet kinematics, $50 \mathrm{GeV}<p_{\mathrm{T}}^{\text {avg }}<63 \mathrm{GeV}$ and $\left|\eta^{\text {dijet }}\right|<0.3$.

The dataset used in this measurement was collected by ATLAS during the $\sqrt{s}=2.76 \mathrm{TeV} p p$ collision datataking in February 2013 at the Large Hadron Collider, with an integrated luminosity corresponding to $4.0 \mathrm{pb}^{-1}$. During data-taking, the mean number of $p p$ interactions per bunch crossing $(\mu)$ varied from 0.1 to 0.5 .

\section{Experimental setup}

The ATLAS detector is described in detail in Ref. [9]. This analysis uses primarily the tracking detectors, the calorimeter, and the trigger system. Charged-particle tracks were measured over the range $|\eta|<2.5$ using the inner detector, which is composed of silicon pixel detectors in the innermost layers, silicon microstrip detectors, and a straw-tube transition-radiation tracker $(|\eta|<2.0)$ in the outer layer, all immersed in a $2 \mathrm{~T}$ axial magnetic field. The calorimeter system consists of a liquid argon (LAr) electromagnetic calorimeter $(|\eta|<3.2)$, a steel/scintillator sampling hadronic calorimeter $(|\eta|<$ 1.7), a LAr hadronic calorimeter $(1.5<|\eta|<3.2)$, and a forward calorimeter $(3.2<|\eta|<4.9)$. The forward calorimeter is composed of two modules situated at opposite sides of the interaction region which provide the $\sum E_{\mathrm{T}}$ measurement.

Data were acquired for this analysis using a central jet trigger covering $|\eta|<3.2$ with multiple jet $p_{\mathrm{T}}$ thresholds, ranging from $40 \mathrm{GeV}$ to $75 \mathrm{GeV}$.

\section{Monte Carlo simulation}

Monte Carlo (MC) simulations of $\sqrt{s}=2.76 \mathrm{TeV}$ $p p$ hard-scattering events were used to understand the performance of the ATLAS detector, to correct the measured $\sum E_{\mathrm{T}}$ and dijet kinematic variables for detector effects, and to determine the systematic uncertainties on the measurement. The PүтнIA 6 generator [10] with parameters chosen to reproduce data according to the AUET2B tune [11] and CTEQ6L1 parton distribution function (PDF) set [12] , the РутніA 8 generator [13] with the AU2 tune [14] and CT10 PDF set [15] and the HeRwIG generator [16] with the UE-EE-3 tune [17] and CTEQ6L1 PDF set were used to generate event samples with the leading jet $p_{\mathrm{T}}$ in the range from $20 \mathrm{GeV}$ to $1 \mathrm{TeV}$. The generated events were passed through a full Geant 4 simulation $[18,19]$ of the ATLAS detector under the same conditions present during data-taking. The simulated events included contributions from additional $p p$ interactions in the same beam crossing (pileup) similar to that in data.

\section{Event reconstruction}

In the offline analysis, charged-particle tracks were reconstructed in the inner detector with an algorithm used in previous measurements of charged-particle multiplicities in minimum-bias $p p$ interactions [20]. Analysed events were required to contain a reconstructed vertex, formed by at least two tracks with $p_{\mathrm{T}}>0.1 \mathrm{GeV}$. The contribution from pileup interactions was suppressed by rejecting events containing two or more reconstructed vertices which each had five or more associated charged-particle tracks.

The jet reconstruction and associated background determination procedures closely follow those developed within ATLAS for jet measurements in heavy ion and $p p$ collisions [1,21].

The $\sum E_{\mathrm{T}}$ quantity was evaluated by measuring the sum of the transverse energy in the cells in one forward calorimeter module $\left(\sum E_{\mathrm{T}}^{\text {raw }}\right)$. The energy signals from the cells were included in the sum without requiring an energy threshold. This quantity was corrected eventby-event to account for detector response, using a calibration procedure derived in simulation, and provides 
an estimate $\left(\sum E_{\mathrm{T}}^{\text {calib }}\right)$ of the full energy deposited in the calorimeter. PYтніA 8 was found to give the best overall description of $\sum E_{\mathrm{T}}$ production and its dependence on the dijet kinematic variables in data.

\section{Data analysis}

In the offline analysis, the leading jet in each event was required to match to a jet reconstructed at the trigger stage. For each leading jet $p_{\mathrm{T}}$ interval, only the trigger with the highest luminosity which was simultaneously $>99 \%$ efficient within that interval was used. The contribution from each event to the $\sum E_{\mathrm{T}}$ measurement was weighted by the inverse luminosity of the trigger used to select it. Events with two jets were selected, where the transverse momenta of the jets were $p_{\mathrm{T}, 1}>50 \mathrm{GeV}, p_{\mathrm{T}, 2}>20 \mathrm{GeV}$, and $p_{\mathrm{T}}^{\text {avg }}>50 \mathrm{GeV}$. The jets were required to have $\eta_{1,2}>-2.8$ to separate them by 0.4 units in pseudorapidity from the $\sum E_{\mathrm{T}}$ measuring region. The leading jet was also required to have $\eta_{1}<3.2$ to match the acceptance of the central jet trigger. Bin migration in the kinematic variables was corrected by applying a multiplicative factor to the results which was derived in simulation from the ratio of the $\left\langle\sum E_{\mathrm{T}}^{\mathrm{gen}}\right\rangle$ evaluated as a function of the dijet variables evaluated with reconstructed to that evaluated with the jets at the particle level.

For each dijet selection, the $\left\langle\sum E_{\mathrm{T}}\right\rangle$ was determined both for the nominal kinematic variables and for the variables in which the jet $\eta_{1,2}$ were flipped, and the other forward calorimeter side was used to measure the $\sum E_{\mathrm{T}}$. The two results were averaged to form the final result.

\section{Systematic uncertainties}

The $\sum E_{\mathrm{T}}$ calibration procedure is susceptible to uncertainties on the overall energy scale of the forward calorimeter, on the amount of material upstream of the calorimeter, on the physics model used to derive the calibration, and on the modeling of pileup in simulation. To evaluate the sensitivity to the physics model, $\sum E_{\mathrm{T}}$ calibrations were derived using simulated PүтніА 6 and Herwig events and compared to that derived using Pythia 8. The uncertainty on the modeling of the pileup within the simulation was determined to be $2 \%$. An additional uncertainty arising from possible defects in the performance of the $\sum E_{\mathrm{T}}$ calibration was obtained from checking the closure of the calibration procedure. A conservative symmetric uncertainty of $5 \%$ was chosen to enclose the potential differences of the closure values from unity observed in simulation.
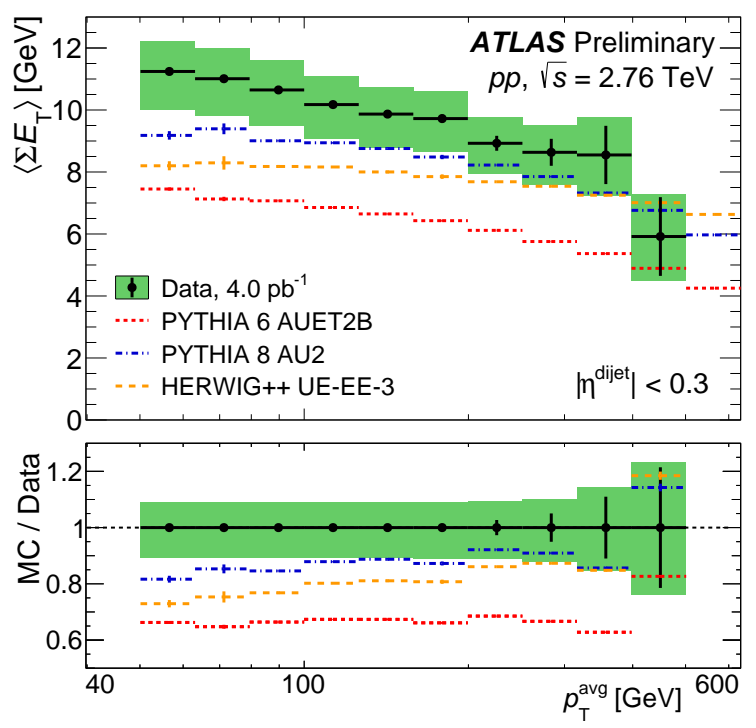

Figure 1: Measured $\left\langle\sum E_{\mathrm{T}}\right\rangle$ in hard-scatter $p p$ collisions, shown as a function of $p_{\mathrm{T}}^{\text {avg }}$ for $\left|\eta^{\text {dijet }}\right|<0.3$ and in comparison with the prediction of three MC event generators. Shaded bands represent the systematic and statistical uncertainties added in quadrature while vertical bars represent statistical uncertainties only. The bottom panel shows the ratio of the three MC generators to the data. From Ref. [6].

For most of the kinematic range except at high- $p_{\mathrm{T}}^{\text {avg }}$ or high $x_{\text {proj }}, x_{\text {targ }}$, the statistical uncertainties are negligible with respect to the systematic ones. The dominant uncertainties on the $\left\langle\sum E_{\mathrm{T}}\right\rangle$ measurement are from the energy scale, the physics model, and the variation of the $\sum E_{\mathrm{T}}$ response with dijet kinematics. The total uncertainty is $+9 \% /-11 \%$ and varies only weakly with selections on dijet kinematics. The uncertainty on the $\left\langle\sum E_{\mathrm{T}}\right\rangle /\left\langle\sum E_{\mathrm{T}}\right\rangle^{\text {ref }}$ quantity was determined by varying the numerator and denominator according to each source simultaneously to properly account for their cancellation in the ratio. The resulting uncertainty is $\pm 5 \%$, dominated by the variation of the $\sum E_{\mathrm{T}}$ response with kinematics, which by its nature does not cancel in the ratio of $\left\langle\sum E_{\mathrm{T}}\right\rangle$ for different kinematic selections.

\section{Results}

Figure 1 shows the $\left\langle\sum E_{\mathrm{T}}\right\rangle$ as a function of $p_{\mathrm{T}}^{\mathrm{avg}}$ for central jet pairs $\left(\left|\eta^{\text {dijet }}\right|<0.3\right)$. The $\left\langle\sum E_{\mathrm{T}}\right\rangle$ is anticorrelated with the dijet $p_{\mathrm{T}}^{\text {avg }}$, decreasing by $25 \%$ as $p_{\mathrm{T}}^{\text {avg }}$ varies from $50 \mathrm{GeV}$ to $500 \mathrm{GeV}$. While the generators systematically underpredict the overall scale of the $\sum E_{\mathrm{T}}$ production, like the data, the $\left\langle\sum E_{\mathrm{T}}\right\rangle$ is generally anticorrelated with $p_{\mathrm{T}}^{\mathrm{avg}}$ in each one.

Figure 2 shows the ratio $\left\langle\sum E_{\mathrm{T}}\right\rangle /\left\langle\sum E_{\mathrm{T}}\right\rangle^{\text {ref }}$ as a function of $p_{\mathrm{T}}^{\mathrm{avg}}$ for different selections on $\eta^{\text {dijet }}$. As the dijet 


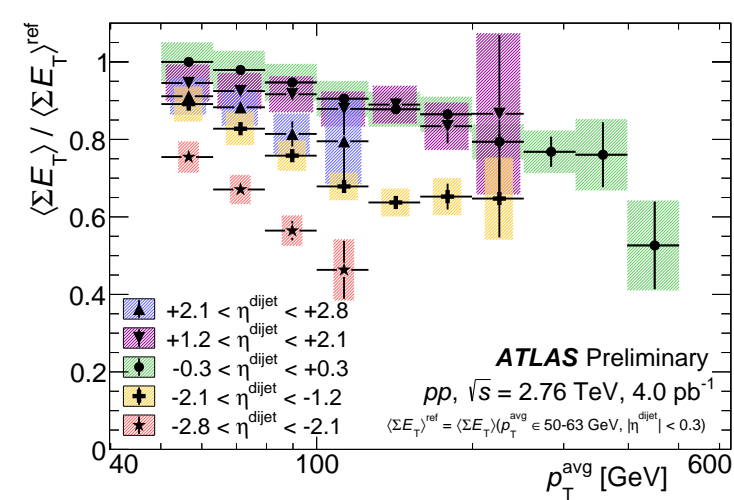

Figure 2: $\left\langle\sum E_{\mathrm{T}}\right\rangle /\left\langle\sum E_{\mathrm{T}}\right\rangle^{\text {ref }}$ in $p p$ collisions, shown as a function of $p_{\mathrm{T}}^{\mathrm{avg}}$ for different selections on $\eta^{\text {dijet }}$. Shaded bands represent systematic and statistical uncertainties, added in quadrature, while vertical error bars represent statistical uncertainties. From Ref. [6].

pair pseudorapidity moves to negative rapidities close to the $\sum E_{\mathrm{T}}$ measuring region (in the target proton-going direction), this anti-correlation becomes stronger and the overall level of the $\sum E_{\mathrm{T}}$ decreases.

To better understand which variables control the $p_{\mathrm{T}}$ and $\eta$ dependence, $\left\langle\sum E_{\mathrm{T}}\right\rangle$ was also studied as a function of $x_{\text {proj }}$ and $x_{\text {targ. }}$. Figure 3 shows the ratio $\left\langle\sum E_{\mathrm{T}}\right\rangle /\left\langle\sum E_{\mathrm{T}}\right\rangle^{\text {ref }}$ as a function of each variable $\left(x_{\mathrm{targ}}\right.$ on the left, and $x_{\text {proj }}$ on the right), while integrating over the other. The value of $\left\langle\sum E_{\mathrm{T}}\right\rangle$ is largely insensitive to $x_{\text {proj }}$ (which corresponds to the Bjorken- $x$ in the proton moving to positive rapidity), changing by only $10 \%$ over the entire range $0<x_{\text {proj }}<1$. On the other hand, $\left\langle\sum E_{\mathrm{T}}\right\rangle$ varies strongly with $x_{\mathrm{targ}}$ (which corresponds to the Bjorken- $x$ in the proton moving to negative rapidity), decreasing by more than a factor of two between $x_{\text {targ }}=0$ and 0.9 in an approximately-linear fashion.

\section{Conclusions}

Measurements are presented of the hard scattering kinematics dependence of transverse energy production at large rapidity in $4.0 \mathrm{pb}^{-1}$ of $\sqrt{s}=2.76 \mathrm{TeV} p p$ collision data with the ATLAS detector at the LHC. They demonstrate that the average level of transverse energy production at small angles is sensitive predominantly to the Bjorken- $x$ of the parton originating in the beamproton which is headed towards the energy-measuring region, and is mostly insensitive to $x$ in the other proton. These results provide counter-evidence to claims that the observed centrality-dependence of the jet rate in $p+\mathrm{Pb}$ collisions simply arises from the suppression of transverse energy production at negative rapidity in the hard-scattered $N N$ sub-collision.

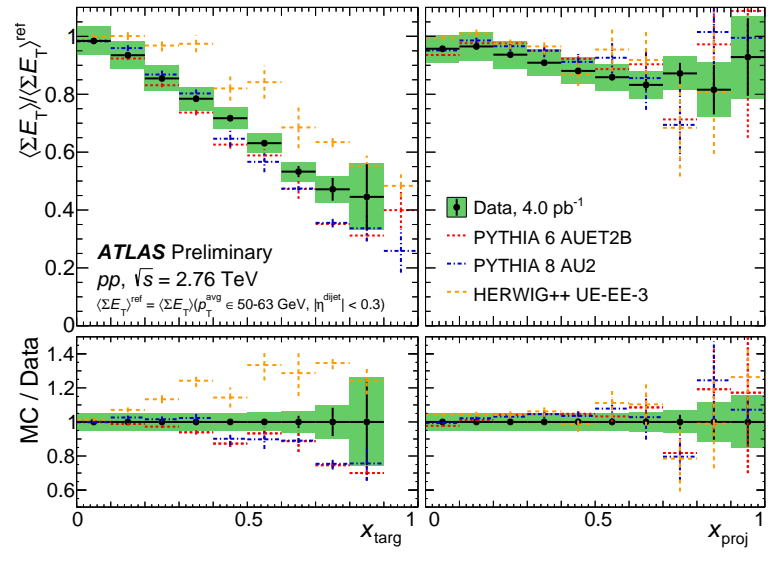

Figure 3: Measured ratio $\left\langle\sum E_{\mathrm{T}}\right\rangle /\left\langle\sum E_{\mathrm{T}}\right\rangle^{\text {ref }}$ in hard-scatter $p p$ collisions, shown as a function of $x_{\text {targ }}$ (left) and $x_{\text {proj }}$ (right). Shaded bands represent total systematic and statistical uncertainties in quadrature while the vertical bars represent statistical uncertainties. The bottom panel shows the ratio of the prediction of three MC generators to the data. From Ref. [6].

\section{References}

[1] ATLAS Collaboration, Phys. Lett. B748 (2015) 392-413. arXiv:1412.4092.

[2] CMS Collaboration, Eur. Phys. J. C74 (2014) 2951. arXiv: 1401.4433

[3] M. Alvioli, et al.,arXiv:1409.7381.

[4] A. Bzdak, V. Skokov, S. Bathe, arXiv:1408.3156.

[5] N. Armesto, D. C. Gulhan, J. G. Milhano, Phys. Lett. B747 (2015) 441-445. arXiv:1502.02986.

[6] ATLAS Collaboration, ATLAS-CONF-2015-019 https ://cds. cern.ch/record/2029360.

[7] M. Cacciari, G. P. Salam, G. Soyez, Eur. Phys. J. C72 (2012) 1896. arXiv:1111.6097.

[8] UA1 Collaboration, G. Arnison et el., Phys. Lett. B136 (1984) 294.

[9] ATLAS Collaboration, JINST 3 (2008) S08003.

[10] T. Sjostrand, S. Mrenna, P. Z. Skands, JHEP 0605 (2006) 026. arXiv:hep-ph/0603175.

[11] ATLAS Collaboration, ATL-PHYS-PUB-2011-009 https : //cds . cern. ch/record/1363300.

[12] J. Pumplin, et al., JHEP 0207 (2002) 012. arXiv:hep$\mathrm{ph} / 0201195$.

[13] T. Sjostrand, S. Mrenna, P. Z. Skands, Comput. Phys. Commun. 178 (2008) 852. arXiv:0710.3820.

[14] ATLAS Collaboration, ATL-PHYS-PUB-2012-003 https : //cds . cern. ch/record/1474107.

[15] M. Guzzi, et al., arXiv:1101.0561.

[16] M. Bahr, et al., Eur. Phys. J. C58 (2008) 639. arXiv:0803.0883.

[17] S. Gieseke, C. Rohr, A. Siodmok, Eur. Phys. J. C72 (2012) 2225. arXiv:1206.0041.

[18] GEANT4 Collaboration, S. Agostinelli et al., Nucl. Instrum. Meth. A506 (2003) 250.

[19] ATLAS Collaboration, Eur. Phys. J. C 70 (2010) 823. arXiv: 1005.4568

[20] ATLAS Collaboration, New J. Phys. 13 (2011) 053033. arXiv: 1012.5104

[21] ATLAS Collaboration, Phys. Lett. B719 (2013) 220. arXiv:1208.1967. 